\title{
Pic, Augustin et Provost, Georges (dir.), Yves Mahyeuc, 1462-1541. Rennes en Renaissance
}

Bernard Merdrignac et Georges Provost

\section{(2) OpenEdition}

1 Journals

\section{Édition électronique}

URL : https://journals.openedition.org/abpo/1870

DOI : $10.4000 /$ abpo. 1870

ISBN : 978-2-7535-1520-8

ISSN : 2108-6443

\section{Éditeur}

Presses universitaires de Rennes

Édition imprimée

Date de publication : 15 décembre 2010

Pagination : 208-211

ISBN : 978-2-7535-1309-9

ISSN : 0399-0826

\section{Référence électronique}

Bernard Merdrignac et Georges Provost, «Pic, Augustin et Provost, Georges (dir.), Yves Mahyeuc,

1462-1541. Rennes en Renaissance », Annales de Bretagne et des Pays de l'Ouest [En ligne], 117-4 | 2010, mis en ligne le 17 janvier 2011, consulté le 22 juillet 2022. URL : http://journals.openedition.org/abpo/ 1870 ; DOI : https://doi.org/10.4000/abpo.1870 
PIC, Augustin et Provost, Georges (dir.), Yves Mahyeuc, 1462-1541. Rennes en Renaissance, Rennes, PUR, 2010, 365 p.

Originaire de Plouvorn en Léon, le Dominicain Yves Mahyeuc a été promu évêque de Rennes en 1507 sur les instances d'Anne de Bretagne dont il était le confesseur. L'austérité de son mode de vie et la charité envers les déshérités dont il témoigna durant son épiscopat lui ont valu de laisser à sa mort en 1541 une "réputation de sainteté ». Au XVII e siècle, l'évêque Pierre de Cornulier (1619-1639) fit procéder en 1637 à un procès informatif sur " les vertus et les miracles » de son prédécesseur à la requête du chapitre cathédral et du couvent dominicain de Bonne-Nouvelle. Complété en 1684-1685 par une trentaine de témoignages supplémentaires "sur ses miracles", l'ensemble fut adressé à Rome à la demande des États de Bretagne. La rigidité et la lenteur des procédures canoniques et surtout, sans doute, en l'occurrence, la prolongation du chantier pluriséculaire de reconstruction de la cathédrale inauguré par Yves Mahyeuc peu avant son décès (Daniel Leloup, "Rennes au temps d'Yves Mahyeuc : une ville entre gothique et Renaissance ») n'ont pas permis à ce procès en béatification d'aboutir. Au reste, ces difficultés n'ont rien d'exceptionnel. Sur une quinzaine d'évêque gratifiés d'un culte plus ou moins spontané entre 1430 et 1540, un seul a été béatifié par Rome (Jean-Michel Matz, "sainteté épiscopale en France $x v^{e}$-début $x v I^{e}$ siècle »). Toutefois, comme le souligne pertinemment Georges Provost, l'un des organisateurs du colloque qui s'est tenu à Rennes en novembre 2007, ce sont bien la "permanence discrète » de la fama sanctitatis du "serviteur de Dieu » et sa résurgence périodique dans la mémoire collective rennaise qui ont permis, à l'occasion du cinquième centenaire de sa consécration épiscopale, de réunir Église catholique, collectivités locales, archives, bibliothèques et chercheurs universitaires de tous horizons pour faire le point des recherches sur Yves Mahyeuc dont le présent ouvrage atteste de la fécondité. En effet, si ce personnage, somme toute méconnu, n'a pas livré tous ses secrets, celui-ci sort métamorphosé de la redécouverte dans les archives romaines des pièces de ce procès - dont des extraits sont ici traduits en annexe par Augustin Pic - et de la reprise, sous tous les angles, de documents variés (archives et ouvrages imprimés, mais aussi gravures, manuscrits enluminés), sans oublier la célèbre verrière de l'Annonciation à La Guerche qui comporte le seul portrait exécuté du vivant de l'évêque (Roger Blot, "Yves Mahyeuc et la chapelle de Tous-les-Saints à la collégiale de La Guerche »), non plus que l'unique traité théologique du futur évêque de Rennes à nous être parvenu.

L'un des "scoops » du colloque, pour reprendre une expression de Jean-Marie Le Gall qui s'est chargé d'en tirer les " conclusions », a été la communication du dominicain Bernard Hodel ("Magister Yvo : I'un et l'autre Yves Mahyeuc »). À partir d'un certain nombre de documents nouveaux tirés des actes de convocation de la congrégation de Hollande et des registres des maîtres généraux de l'ordre, cette contribution remet en question la biographie traditionnelle de l'évêque de Rennes fondée sur "La vie du bien-heureux père $f$. Yves Mayeuc » par Albert Le Grand dans ses Vies des saints de Bretagne Armorique (1636). Les incohérences chronologiques quant aux dates d'accès aux grades universitaires et aux responsabilités internes dans l'ordre dominicain conduisent en effet Bernard Hodel à supposer l'existence de deux dominicains homonymes (probablement l'oncle et le neveu) ayant poursuivi des carrières analogues (l'épiscopat excepté). Yves Mahyeuc senior, maître en théologie serait resté confesseur d'Anne de Bretagne jusqu'à la promotion d'Yves Mahyeuc junior sur le siège épiscopal rennais en 1507. Ce dernier aurait succédé dans cette fonction à son oncle après le décès de celui-ci à une date inconnue. Cette hypothèse audacieuse a manifestement conduit plusieurs des contributeurs à nuancer, voire à remanier, leur texte définitif. Ainsi Lionel Rousselot ("La Questio 
perpulchra, une œuvre d'Yves Mahyeuc dédiée à Louise de Savoie ») montre que la teneur de l'unique traité théologique connu du futur évêque de Rennes correspond à « ce que l'on peut attendre d'un confesseur royal ». Mais il fait référence en note aux recherches de Bernard Hodel qui ne permettent pas d'affirmer qu'Yves Mahyeuc exerçait déjà cette fonction lors de la composition de cet ouvrage. En tout cas, il est établi qu'en 1512, c'est bien l'évêque de Rennes qui occupait ce poste. De même, Jean-Christophe Cassard, ("Yves Mahyeuc, dominicain bas Breton devenu évêque de Rennes »), à la suite d'une analyse du récit d'Albert le Grand (à laquelle il procède avec la « prudence » et la "réserve » qui s'imposent) et avant d'aborder l'« ancrage rennais » du prélat d'extraction léonarde, consacre un paragraphe au doute qui " s'est immiscé dans les esprits » à l'occasion du colloque. Tout en admettant qu'un pareil cas de népotisme n'aurait rien d'exceptionnel à l'époque, il relève toutefois, dans les pièces relatives au couvent de Nantes fréquenté par le futur évêque de Rennes, les mentions en tant que prieur et "vicaire substitut de couvents réformés de Bretagne » soit d'Yves "Maieuc », soit d'Yvo Gracilis. Puisque l'adjectif gracilis ne peut être considéré comme la latinisation du patronyme breton Mahyeuc (formé sur I'hagionyme Maioc), ce n'est plus un duo, mais un trio de Dominicains bretons quasiment contemporains, dont Jean-Christophe Cassard propose malicieusement d'envisager l'existence à une génération d'écart. Quant à Bruno Restif (" Un évêque issu de la réforme régulière au temps de la première réforme séculière $»)$, il rappelle d'abord lui aussi la chronologie d'Albert le Grand, tout en faisant état des difficultés que soulève celle-ci. II met en rapport la mention d'Yvo Gracilis, chargé dès 1485 d'introduire la réforme à Auxerre avec celle d'Yves Mahyeuc " confesseur de la reine " nommé en 1504 prieur du couvent provençal de Saint-Maximin pour le réformer, en passant par le "vicaire substitut pour la Bretagne » qui réforme celui de Dinan en 1496 et qui pourrait être l'un ou l'autre (rien n'impliquant d'ailleurs à chaque fois une intervention sur place). Aussi une triple confusion de la part d'Albert le Grand (et des auteurs qui l'ont suivi) paraît-elle « peu plausible » à ce chercheur.

L'objet d'une recension ne saurait être d'apporter une solution définitive à une question "qui fut comme l'événement de ce colloque ", ainsi que le souligne le frère Augustin Pic, dans son "Avant-propos ». On notera cependant que c'est la date de naissance d'Yves Mahyeuc en 1462 fournie par Albert le Grand qui constitue le nœud du problème. D'expérience, le signataire du présent compte-rendu a pu constater pour sa part que les supputations chronologiques de l'hagiographe du xvII siècle sont souvent sujettes à caution. Or Bruno Isbled, en présentant « Yves Mahyeuc à travers les archives rennaises » fait état de la notice rédigée par le jurisconsulte Gilles Becdelièvre (1515-1579) dans le Livre de Raison familial qui enregistre la mort du pieux évêque de Rennes en odeur de sainteté le mardi 21 septembre 1541 (en fait, le 20 septembre) dans la « quatre-vingt-dixième année de son âge » (nonagesimum sue aetatis annum). Certes, on ne peut attendre d'un document du milieu du $\mathrm{xVI}^{\mathrm{e}}$ siècle la précision d'un acte d'état-civil. Mais voici un témoignage contemporain qui, en incitant à antidater d'une dizaine d'années (soit aux environs de 1452) la naissance d'Yves Mahyeuc par rapport à la chronologie communément reçue, est susceptible de lever les contradictions pointées par Bernard Hodel, tout en faisant l'économie des hypothétiques doublures du "bon Yves » (au double sens de l'adjectif) dont celui-ci invite de ce fait à postuler l'existence. En tout état de cause, l'historicité du " jacobin ami de la duchesse et évêque de Rennes de 1507 à 1541 » est incontestable. Les participants au colloque se sont employés à élargir les perspectives d'une biographie spirituelle encore incertaine sur certains points, en replaçant la mémoire de ce personnage dans le contexte historique de son époque, comme l'indique le sous-titre du livre, Rennes en Renaissance. Le plan de l'ouvrage traduit la démarche adoptée avec succès par les initiateurs de la rencontre. Une 
première partie intitulée "La mémoire d'un saint » conduit à une deuxième partie présentant le "Frère prêcheur » qui précède logiquement la troisième consacrée à "L'évêque " pour découvrir enfin "L'homme et son temps" dans la dernière partie. II n'est évidemment ni possible, ni souhaitable de détailler ici la teneur de chacune des études réunies dans ces Actes. On se contentera de mettre l'accent sur les deux termes du sous-titre afin de proposer aux lecteurs des ABPO un aperçu de la richesse de l'ouvrage.

Yves Mayeuc apparaît bien avant tout comme "le saint de la cité de Rennes" (Jean-Marie Le Gall). Gauthier Aubert (« Des Rennais ordinaires : Roch, Perrine, Guillemette et les autres, miraculés d'Yves Mahyeuc ») avance même la formule de " " religion civique ", à caractère identitaire incontestable ». L'étude fine des bénéficiaires de ses miracles par Georges Provost (" Une ville et son saint : Yves Mahyeuc à Rennes - XVII ${ }^{\mathrm{e}}$-xII siècle ») démontre que ceux-ci s'inscrivent dans un cadre essentiellement rennais qui déborde au sud-ouest du diocèse, c'est-à-dire à proximité du manoir épiscopal de Bruz. Du temps du pieux évêque, celui-ci constituait le noyau de la partie rurale de son régaire et parmi les noms des châtelains et receveurs successifs qui tiennent les comptes de la seigneurie, Bruno Isbled relève celui de Jean Mahyeuc, "frère de Monseigneur ». Les archives rennaises laissent en outre percevoir la présence aux côtés de l'évêque d'un petit clan familial originaire de son Léon natal qui l'épaule dans la gestion du diocèse: Pierre Mahyeuc, son neveu; Hervé Mahyeuc, I'un des chanoines les plus assidus du chapitre (en tant que scolastique) qui lui est sûrement étroitement apparenté; Goulven Abguillerm, " écuyer de l'évêque », et son exécuteur testamentaire, qui porte un patronyme typiquement léonard et a pour saint patron le seul saint du Léon vénéré à la cathédrale de Rennes. Effet de sources (exemplaire dans ce cas précis) que la "distorsion", soulignée par Jean-Michel Matz, entre les documents hagiographiques évoquant l'aura du prétendant à la sainteté et les actes de la pratique qui mettent en évidence sa préoccupation d'un gouvernement avisé de la cité épiscopale! Diverses interventions contribuent à préciser le paysage urbain rennais au $x v l^{e}$ siècle. On retiendra les conclusions de Daniel Pichot («Rennes en 1543 : «portrait» d'une ville ») qui commente le "pourtraict de la ville de Rennes» dans le célèbre Manuscrit de la Vilaine, sans doute réalisé par le peintre Olivier Aulion, peut-être sur commande de la municipalité rennaise. L'artiste donne à voir une cité encore profondément médiévale dans sa conception, même si l'affirmation de la bourgeoisie introduit déjà " une note de modernité ». Bref, « les temps nouveaux ne sont pas marqués par une rupture brutale » et Yves Mahyeuc, comme bien d'autres, aura été " I'homme de ce passage ».

On peut donc concéder à Jean-Marie Le Gall que l'évêque de Rennes n'est sans doute pas le prélat le plus « emblématique de la Renaissance ». Toutefois, la complémentarité des travaux des historiens médiévistes et modernistes rassemblés ici confirmerait qu'Yves Mahyeuc est bien un protagoniste actif de la transition entre le Moyen Âge et la Renaissance, "si tant est que ces catégories générales aient un sens » (comme le note au passage Hervé Martin). Partie prenante du « milieu particulier des confesseurs royaux dominicains " (Guy Bedouelle, "Yves Mahyeuc et le milieu humaniste parisien »), celui-ci a des sympathies, en particulier entre 1505 et 1515, pour certains membres (Robert Fortuné, Guillaume Petit, Alphonse Riccius, Geoffroy Boussard) du cercle de Lefèvre d'Etaples (1460-1536) dont il partage - discrètement, il est vrai, « sans jamais apparaître en première ligne »- les aspirations à une réforme de l'Église par la piété, le retour aux sources et l'érudition. Il est en relations avec Jean Clérée (1455-1507) confesseur de Louis XII, puis maître général de l'ordre dominicain, qui est l'un des prédicateurs à succès de cette époque. "Confesseur, et a fortiori confesseur royal, rimait alors avec prédicateur ». Même si 
aucun de ses sermons n'a été conservé, on peut être assuré qu'Yves Mahyeuc a certainement prêché. C'est pourquoi Hervé Martin ("Quelques réflexions sur l'état de la pédagogie religieuse vers $1500 »)$ analyse une série de sermons de Carême de son ami Jean Clérée pour mettre en évidence l'efficacité de ces « fruits d'arrière-saison de la machine à prêcher scolastique », revitalisés par la recherche de l'« effet de réel » et la spontanéité des dialogues, anticipant sur " la comédie de mœurs de l'époque moderne ». Yves Mahyeuc était lui aussi pleinement rompu à la méthode scolastique comme le montre l'analyse par Lionel Rousselot de cette Questio perpulchra que l'évêque de Rennes a ressorti de ses dossiers (questionem quam inter cartas et membranas habebas) pour la communiquer à son collègue Alphonse Riccius, confesseur dominicain des officiers du roi, qui la fait imprimer en 1513 pour la gouverne de Louise de Savoie, mère du futur François $\left.\right|^{\text {er }}$. La maîtrise des techniques scolastiques de la "question disputée » n'exclut bien sûr aucunement des préoccupations humanistes de la part de l'auteur de ce traité sur le thème de la Fortune. II convient donc de se garder ici de toute opposition caricaturale entre "sorbonagres " rassis et théologiens novateurs dans la ligne de Lefèvre d'Etaples et d'Erasme, à plus forte raison s'il s'agit d'un exercice scolaire rédigé dans la jeunesse de l'auteur, comme le suggère Lionel Rousselot, " pour la conquête d'un grade universitaire ». Le même paradoxe se relève dans ce que l'on peut percevoir du rapport d'Yves Mahyeuc au livre (Sarah Toulouse, « À la recherche de la bibliothèque d'Yves Mahyeuc. Les Dominicains de Rennes et les livres au Xvie siècle »). Cet amateur de manuscrits enluminés à l'instar de sa royale patronne, est cependant responsable de l'implantation pérenne de l'imprimerie à Rennes - même si un atelier éphémère est mentionné en 1484 - afin de publier le Liber Marbodi, recueil des poèmes de Marbode († 1123), I'un de ses lointains prédécesseurs sur le siège épiscopal de Rennes (Malcolm Walsby, "Yves Mahyeuc, Jean Baudouyn et l'implantation de l'imprimerie à Rennes »). Ce choix est révélateur. En effet, l'œuvre poétique de Marbode est I'un des fleurons de la "Renaissance du XII siècle ». Dès lors, l'Église ne présente plus seulement ce bas monde comme " une vallée de larmes » et déjà point l'espoir d'un futur que l'ancien maire de Rennes Edmond Hervé, dans son discours d'ouverture du colloque, semblait pourtant considérer - avec « beaucoup de précautions » (au moins rhétoriques) - comme spécifique de la Renaissance du $x v l^{e}$ siècle, puis du Temps des Lumières. Le Moyen Âge n'est-il pas, somme toute, qu'un enchaînement de renaissances?

Témoin privilégié de ce glissement de l'époque médiévale à la Renaissance proprement dite, Yves Mahyeuc apparaît aussi (à condition de restituer au personnage son unicité mise à mal par l'hypothèse avancée par Bernard Hodel) comme un des principaux acteurs du demi-siècle au cours duquel le duché de Bretagne était en passe de devenir une province du royaume de France. En tant que directeur de conscience de la jeune duchesse Anne, il aurait contribué, en 1491, comme le rappelle Jean-Christophe Cassard, à dissiper chez sa dévote pénitente les scrupules que son précédent mariage par procuration avec Maximilien suscitait à l'encontre son union avec le roi Charles VIII. En tant qu'évêque de Rennes, il préside en 1532 au couronnement ducal du dauphin François III dans sa cathédrale (Philippe Hamon, «Rennes, 1532 : le dernier couronnement ducal»). 